\title{
Trends in maternal and child health outcomes in a health systems intervention: a case of Obekai dispensary in western Kenya
}

\author{
Fabian Esamai ${ }^{1}$, Ann Mwangi ${ }^{2}$, John Tabu ${ }^{3}$, Mabel Nangami ${ }^{4}$, Edwin Were ${ }^{5}$, David Ayuku ${ }^{6}$ \\ 1 Department of Child Health and Paediatrics, School of Medicine College of Health Sciences, Moi University, Eldoret, Kenya, ${ }^{2}$ Department of \\ Behavioural Sciences, School of Medicine College ofHealth Sciences, Moi University, Eldoret, Kenya, ${ }^{3}$ Department of Disaster Risk Management, \\ School of Public Health, College of Health Sciences, Moi University, Eldoret, Kenya, ${ }^{4}$ Department of Health Management and Health Policy, Schoolof \\ Public Health, College of Health Sciences, Moi University, Eldoret, Kenya, ${ }^{5}$ Department of Reproductive Health, School of Medicine College of Health \\ Sciences, Moi University, Eldoret, Kenya, ${ }^{6}$ Department of Behavioural Sciences, School of Medicine College of Health Sciences Moi University, Eldoret, \\ Kenya \\ Keywords: enhanced health care, neonatal health, maternal health, health system \\ https://doi.org/10.29392/001c.12840
}

\section{Journal of Global Health Reports}

Vol. 4, 2020

\section{Background}

Maternal, fetal and neonatal mortality are higher in low-income compared to high-income countries primarily due to weak health systems that impede access and utilization of health services. Despite significant improvements in maternal, neonatal and under five children indicators, in some low-income countries, including Kenya these indicators remain relatively high prompting the search for innovative interventions to catalyze the progress towards attaining the Sustainable Development Goal (SDG) 3 target by 2030. We describe the results of a study that assessed the impact of an innovative health systems approach on maternal, neonatal and under-five children outcomes.

\section{Methods}

This was a four year pre-post prospective study to describe trends in the outcomes through the implementation of the Enhanced Health Care (EHC) using the Find Link Treat and Retain (FLTR) strategy. This was implemented in the catchment population of a 'level 2' facility, Obekai dispensary in Busia County, Kenya between January 2016 and January 2019. The study population was pregnant women, newborns and under-five children identified and referred to the facility over the study period. Women were identified in the community by community health workers early in pregnancy and followed up in Obekai dispensary until delivery. The newborns were followed up for the whole period the mother baby dyads were in the study. The EHC was implemented on these pairs during the duration of study upto 2-3 years. An interrupted time series model for a single group was used to assess the effect of the intervention on the outcome.

\section{Results}

Attendance in the outpatient, under five and antenatal clinic increased by $76 \%, 37 \%$ and $54 \%$ respectively from 2015 to 2018 . There was a $90 \%$ fully immunization coverage with 97\% and 94\% BCG and Polio coverage respectively among children studied. There was a 91\% facility delivery rate among the pregnant women enrolled in the study. After introduction of FLTR the immunization uptake increased significantly per quarter at a rate of 29.2 (95\% confidence interval, $\mathrm{CI}=20.1-38.3)$. There was an increase in facility delivery, antenatal (ANC) attendance and decrease in neonatal death after introduction on FLTR.

\section{Conclusions}

Maternal and under-five health indicators in Obekai improved over the study period following the implementation of the EHC package.

Most countries in sub-Saharan Africa have weak health systems in terms of low funding, operational and management inefficiency, poor quality of health services, inequities in distribution of the health workforce, and low capacity for planning, budgeting, and governance. ${ }^{1}$ Some of the conse- quences of the weak health system include rising disease burden including non-communicable diseases, new and reemerging epidemics. Specifically, most countries in sub-Saharan Africa did not meet MDG goals 4 and 5 because of weak health systems. ${ }^{2}$ Building health systems that are re- 
sponsive to health needs of populations requires innovative application of technologies and human resources with the capacity to conduct research and use evidence to inform health policy making and practice.

Health systems research (HSR), is an evolving field of study that "is interdisciplinary in nature and aims to provide policy and practice relevant information that improves the health system as a whole by addressing the goals of equity, efficiency, effectiveness, and sustainability, ultimately leading to improved health status". 3 The high maternal and child morbidity and mortality in Africa is testament to nonresponsive health systems that perpetuate inequalities due to ineffective and unsustainable interventions.

Studies and reports from low and high-income countries indicate that maternal, fetal and neonatal mortality are higher in many low-income compared to high-income countries. The reasons for these discrepancies are many including little prenatal care and home deliveries.

In low-income settings, maternal mortality rates range from 150 to more than 1000 per 100,000 live births ${ }^{1}$ while rates of stillbirth and neonatal mortality generally range from 20 to 40 per 1000 births. ${ }^{4}$ Intrapartum stillbirth, or those stillbirths that occur during labor and delivery, are an important indicator of the quality of obstetric care. ${ }^{4-6}$ While in high-income countries, intrapartum stillbirths have nearly been eliminated, in low-resource settings, up to half of all stillbirths occur in the intrapartum period.

Universal access to high quality facility care substantially reduces mortality and morbidity. ${ }^{7}$ Poor quality of antenatal care results in failure to detect and manage high risk pregnancies in low- and middle-income countries. This poor access to antenatal care and the obstetric complications contribute to high early neonatal deaths including stillbirths. In high-income countries, access to prenatal care is nearly universal.

In the neonatal period prematurity, infections and asphyxia account for about $80 \%$ of all deaths worldwide with most of these occurring in developing countries. The contributing factors to asphyxia, preterm births and neonatal sepsis are predominantly antenatal and intra-partum. Four in ten under-five deaths occur during the first month of life. Among children who survive beyond the first month, pneumonia, diarrhea and malaria are the leading killers with infectious diseases. Malnutrition also accounts for over two thirds of these deaths as a co-morbidity. Worldwide, more than one-third of all under-five deaths are associated to malnutrition. $1,8,9$

In developing countries, a significant proportion of deliveries occur outside health facilities and by unskilled birth attendants. Facility delivery is known to be the single most effective intervention for preventing maternal and neonatal morbidity and mortality. However, this is only true if there is provision of quality care at the facility. In Kenya, about $61 \%$ of deliveries occur in health facilities and only $7 \%$ of newborns are resuscitated by skilled health workers trained on neonatal resuscitation and only $22 \%$ are born in health facilities with appropriate resuscitation equipment. ${ }^{10-14}$

Since 2000, most countries have focused on interventions that aim to increase health facility delivery and neonatal and early childhood services - high impact interventions for MNCH. Evidence is emerging that during the same period health sector reforms in most Sub-Saharan countries, including Kenya, stalled and health systems have remained weak as characterized by inadequate funding , inefficient resource management and poor policy implementation. ${ }^{15}$

One emerging challenge to achieving and sustaining desired targets for maternal and child health is neglect of the gap between the community and primary care health facilities, failure to espouse a systems/holistic approach as well as the continuum of the three delays model. ${ }^{16}$ Although evidence is generally weak on maternal health indicators, information from KDHS $2003^{17}$ showed that maternal, neonatal and child health indicators had either stalled or were worsening - primarily due to poor quality of care at health facility level (supply) and poor physical, financial and psychological access (demand) to reproductive health services. In 2006, the government formally recognized the importance of closing the gap (interface) between the community and front line facilities by launching the Community Health Strategy. ${ }^{18-20}$ However, some of the promising interventions have largely been small scale and unsustainable limiting the opportunities for replication and scale up.

This study addressed these poor indicators in maternal and child health at dispensary level by implementing an enhanced health care (EHC) program through a "find link treat and retain" (FLTR) strategy with the application of the World Health Organization (WHO) pillars of the health system. The intervention implemented was the EHC package, which was implemented through the FLTR strategy, which comprised of the elements outlined in the methods section below. The aim of this paper is to describe the trends in maternal, newborn and child health outcomes during the study period.

\section{METHODS}

This was a prospective (pre and post)study carried out over a four-year period. In the first year (2015) baseline data was collected followed by implementation of the study package (post-intervention), of the EHC through the FLTR strategy for a period of 3 years (2016-2018). The study was carried out in Obekai dispensary; a level 2 facility which comprises the primary care level within Kenya's health system. Obekai dispensary is located in Kaliwa location in Busia County. Kaliwa location has a population of about 10,000 people which is about $1.2 \%$ of the population of the County. This was part of a larger prospective quasi-experimental comparative study which has been published and described. ${ }^{21,22}$

The CHWs were taken through the WHO training modules on the danger signs of pregnant mothers and newborns and the general guidelines on the responsibilities of the Community Health worker (CHWs). These trainings were conducted at the baseline in 2015 and repeated as refresher courses in 2016, 2017 and 2018 with updates provided.

The skilled health workers (SHWs), who were nurses, and a clinical officer went through the protocol training, the Basic and Comprehensive emergency on maternal and neonatal care courses (BeMONC and CeMONC) course in 2015 and repeated annually in 2016, 2017 and 2018 including updates on new developments.

The trainings for SHWs and CHWs included strategies for 
community entry and disease surveillance.

\section{STUDY POPULATION}

The study population was all pregnant women and children under five years of age living in the catchment area of Kaliwa location, which is the catchment population of Obekai dispensary during the study period. These were 537 women and baby dyads over the study period.

\section{INCLUSION CRITERIA}

We included: i) all pregnant women in the study cluster who gave written consent; and ii) all newborn babies, infants and under five children born during the life of the study within the study population.

\section{FLTR STRATEGY}

The EHC package (Box 1) was implemented using the FLTR strategy to improve access and quality of care of maternal and child health at levels 1 and 2 . The details of the methods applied have been described elsewhere. ${ }^{21}$ This included identification of pregnant mothers early in pregnancy by community health workers (CHWs) and the implementation of the 10 elements of the EHC to each one of them and to their babies. These mothers were taken through a birth preparedness exercise early in pregnancy with emphasis on antenatal care visits (ANC), saving money for delivery and hospital care after delivery, planning for transport to the facility for delivery, identifying a delivery facility and delivering in the facility. Most of the elements of the EHC were implemented during the ANC visits, which were at least four visits in a pregnancy with the aim of reaching eight visits as approved by the WHO. The children were followed per the immunization schedule as well as follow up in the community for any danger signs.

\section{Box 1. The Enhance Health care (EHC) components}

Early identification and surveillance of the pregnant woman and newborn babies.

Recognition and management of pregnancy-related complications, particularly pre-eclampsia, haemorrhage, premature rupture of membranes and infection.

Recognition and treatment of underlying or concurrent illness in pregnancy - malaria, anaemia, infection among others.

Screening for conditions and diseases such as syphilis, HIV infection and hepatitis B.

Preventive measures through administration of tetanus toxoid, immunisation, de-worming, iron and folic acid supplementation, intermittent preventive treatment of malaria in pregnancy (IPT), prenatal vitamins provision and use of Long Lasting Insecticides Treated Nets (LLITNs). Developing a birth and emergency preparedness plan.

Immunization of all young infants and underfive children born during the study period including Vitamin A administration at 6 months of age and yearly.

Obtain weights for all newborns, infants, under five children and pregnant mothers in the community and health facilities by providing weighing scales (infant, adult).

Malaria diagnosis using the rapid diagnostic tests in the community and health facility.

Haemoglobin estimation using Haemocue in the community and at the facility.

We applied the FLTR (Find, Link, Treat, and Retain) strategy to support a successful implementation of Enhanced Health Care (EHC). This required direct investment in health system elements of the WHO which includes leadership and governance; human resources; medical products, vaccines and technologies; and health services delivery; HMIS, health financing and infrastructure. All these elements were implemented in the study to achieve the objectives of the study and formed the principles under which the study was formulated and applied.

Find - involved investment in the human resources for health (HRH) component of the health system by influencing recruitment of adequate and competent CHWs from within intervention villages. It also involved investment in improvements in management components through strengthening of supervision by trained health workers who included a clinical officer and three nurses. The CHWs implemented the EHC using appropriate technologies and supplies (computers, HMIS, mobile phone and household registers) to improve efficiency and strengthen the health information system. The carried out surveillance strategies to identify (FIND) pregnant women early in pregnancy preferably within the first trimester.

Link - CHWs encouraged women to come for prompt 
antenatal care and maternal and child health (ANC/MCH) clinic attendance at the dispensary and scheduled ANC/ $\mathrm{MCH}$ clinic appointments by mobile phone device at the time of initial CHW visit (Link). This links directly to efficiency in Service delivery, investment in technologies (mobile phone) and health information for efficient management of referrals. This created a link between level 1 and level 2 of the health system.

Treat - The ANC/MCH clinics in Obekai dispensary provided the complete EHC package to each subject to address the quality of service delivery, technologies and commodities component. These included haemoglobin testing using the haemocue machine and malaria test using the Rapid Diagnostics Tests (RDTs). Any ailments were treated at the facility and where referral was needed they were referred to the higher level facility. Newborns were given the routine vaccinations, weights taken and prenatal and vitamin $\mathrm{A}$ provided including all the relevant services as outlined in the EHC package. The services were offered by the Clinical Officer, 3 nurses and a laboratory technician located at the dispensary. This also included the County health staff posted to the facility who were two nurses in addition to the study clinical officer, nurse and laboratory technician.

We provided essential equipment, drugs and dressings as per the essential commodities list of the Ministry of Health for the level 2 facility.

We utilized the Ministry of Health approved standards of care for this level of care for the management of illnesses. We provided for acceptable waiting time for patients as recommended and conducted exit surveys for all mothers who were treated at this facility as they left the facility on the same day.

Retain - Subjects were tracked and if one defaulted the CHW immediately followed up the subject at home to counsel the woman/family and accompany the woman to the health facility for the ANC/MCH visit. This component of the intervention links to building efficient and effective health information system that allows for efficiency in monitoring/tracking of subjects at antenatal, delivery and postnatal care. The component used cost-reduction in tracking by using mobile phones. The follow up therefore ranged from 6 months to 3 years depending on the month and year of birth over the three-year study period. This translates into the retention of the women and children in the health system throughout the period of the study. This FLTR strategy only applied to enrolled pregnant mothers and their infants within the intervention location. Women who became pregnant for the second time during the study period were enrolled and followed up as per protocol. There were therefore cohorts of varying follow up periods ranging from 6 months and 3 years depending on when they are enrolled in the study.

\section{DATA MANAGEMENT AND ANALYSIS}

Data were collected using questionnaire that were designed to capture the information in the Mother Child Card. The data was entered in Microsoft Access Database in a computer in the facilityby a data entry clerk. Regular data quality checks were done by the Data manager. Data was then exported to STATA Version 15 for analysis. Descriptive statistics such as frequencies and percentages were used for categorical variables. We used plots to describe the trends in uptake of various MCH elements over time. Time trend analysis was used to compare the trend before and after the intervention. To assess the effect of the intervention we used an interrupted time series model for a single group to assess the effect of the intervention. We captured data every month during the study period, we aggregated data at each quarter and we therefore had four measurements before intervention and twelve measurements after intervention and thus we could assess the trend before and the trend after intervention. The models were fitted in STATA for the different maternal and neonatal outcomes.

\section{ETHICAL CONSIDERATIONS}

The study was approved by the Institutional Research and Ethics Committee of Moi University and the County Government of Busia Ministry of Health. All participating women were enrolled after obtaining a written consent by the skilled health workers in the dispensary.

\section{RESULTS}

Overall there were 537 women enrolled in the study and 442 (82.3\%) were consistently followed up for the 3 years on monthly basis at the Obekai facility. During the monthly revisits, there were over 1940 revisits expected in 2018 out of which 1775 (92\%) were achieved by the enrolled women. There were 34 referrals to higher level facilities from Obekai dispensary out of which 30 (88.2\%) complied with the referral and the reverse referral back to Obekai from the higher facility.

\section{GENERAL CLINIC ATTENDANCE, UNDER FIVE AND ANC ATTENDANCE}

The trends in attendance for the general clinic, child welfare clinic and antenatal clinic attendance at baseline in 2015 to 2018 show a marked increase as shown in Table 1 and Figures 1-4. Table 1 shows the increase in clinic attendance for both adults and children from 2015 at baseline to 2018 during the intervention. There was a marked increase when compared with the projected increase as per the dispensary's annual development plans. This table also shows the antenatal attendance trends during the study period. 
Table 1. Trends in general, under five and ANC attendance at Obekai dispensary over the study period

for all patients

\begin{tabular}{|c|c|c|c|c|c|c|c|c|c|c|}
\hline \multirow[t]{2}{*}{ Year } & \multirow{2}{*}{$\begin{array}{l}2015 \\
\text { Baseline }\end{array}$} & \multicolumn{3}{|c|}{2016} & \multicolumn{3}{|c|}{2017} & \multicolumn{3}{|c|}{2018} \\
\hline & & Actual & Projected & $\begin{array}{l}\text { \% change from } \\
\text { baseline }\end{array}$ & Actual & Projected & $\begin{array}{l}\text { \% change from } \\
\text { baseline }\end{array}$ & Actual & Projected & $\begin{array}{l}\text { \% change from } \\
\text { baseline }\end{array}$ \\
\hline Facility general attendance & 3073 & 4825 & 2190 & 57 & 4662 & 2190 & 52 & 5409 & 2502 & 76 \\
\hline $\begin{array}{l}\text { Under five outpatient } \\
\text { attendance }\end{array}$ & 2190 & 2890 & 1530 & 32 & 2809 & 1548 & 28 & 3011 & 1788 & 37 \\
\hline Antenatal clinic attendance & 506 & 757 & 336 & 50 & 809 & 336 & 60 & 777 & 366 & 54 \\
\hline
\end{tabular}




\section{ANC ATTENDANCE}

The trend in ANC attendance is shown in Figure 1. We observed that the estimated number of women who attended ANC in the first quarter was 140.2, the number increased every quarter prior to the intervention by 0.5 though not statistically significant ( $P=0.978,95 \%$ confidence interval, $\mathrm{CI}=-38.9-39.9)$. In the first quarter immediately after the intervention there was an increase in ANC attendance by 17.2 though not statistically significant followed by an increase in ANC attendance per quarter relative to the pre-intervention trend of 4.0 though not statistically significant ( $P=0.832$, 95\% CI $=-36.3-44.3)$. We observed that after introduction of FLTR the ANC attendance increased per quarter at a rate of $4.5(95 \% \mathrm{CI}=-3.9-12.9)$.

\section{IMMUNIZATION UPTAKE}

We observed that the estimated number of children who were immunized in the first quarter was 87.7, the number decreased significantly every quarter prior to the intervention by $24(P<0.001,95 \% \mathrm{CI}=-34.6-13.3)$. In the first quarter immediately after the intervention there was a significant increase in the number of children immunized by 63.4 , followed by a significant increase in immunization uptake attendance per quarter relative to the pre-intervention trend of 53.2 (P-value<0.001, 95\%CI [40.8, 65.6]). We observed that after introduction of FLTR the immunization uptake attendance increased significantly per quarter at a rate of 29.2 (95\% CI=20.1-38.3). These results are shown in Figure 2.

Facility delivery

The number of women delivering in the facility over time is shown in Figure 3. From the figure we observed that in the first quarter prior to the intervention the estimated number of women who delivered in a facility was 89.2 , this increased significantly per quarter by 18.5 ( $p$-value $<0.001$ ). Immediately after the intervention in the first quarter the estimated number of hospital deliveries decreased by 5.1 though not statistically significant $P=0.568$, this was followed by a statistically significant decrease relative to the pre intervention trend of $15.3(P<0.001)$. Overall we observe that after introduction of FLTR the number of facility deliveries increased by 3.2 though not statistically significant, $P=0.055$.

\section{NEONATAL DEATH}

In the first quarter prior to the intervention the estimated number neonatal deaths were 4.7 , this decreased significantly per quarter by $1(P<0.001)$. Immediately after the intervention in the first quarter the estimated number of hospital deliveries decreased by 0.2 though not statistically significant $P$-value $=0.836$, this was followed by a statistically significant increase relative to the pre intervention trend of 0.9 (p-value<0.001). Overall we observe that after introduction of FLTR the number of facility deliveries decreased by 0.1 though not statistically significant $P=0.473$. The results are shown in Figure 4.

\section{MATERNAL AND CHILDHOOD OUTCOMES}

Table 2 shows the maternal outcomes from baseline in 2015 to the end of the intervention in 2018. We observed that the

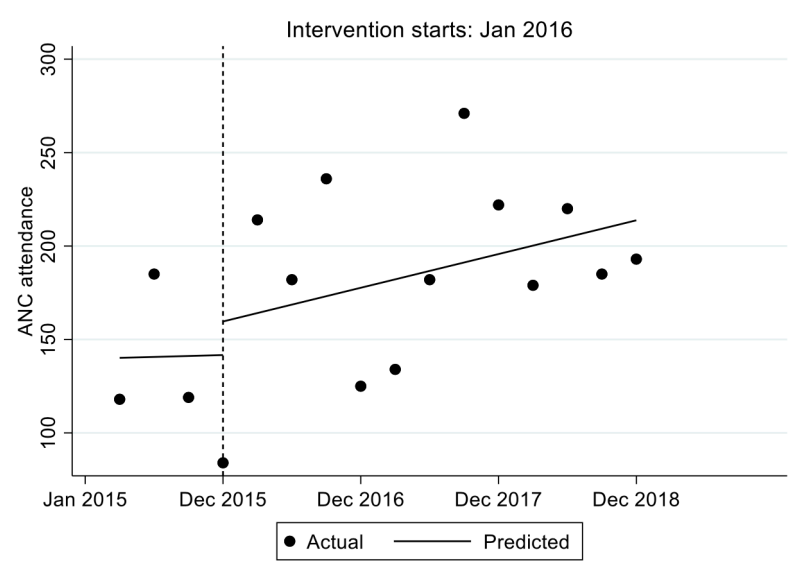

Figure 1. Trend in antenatal care attendance.

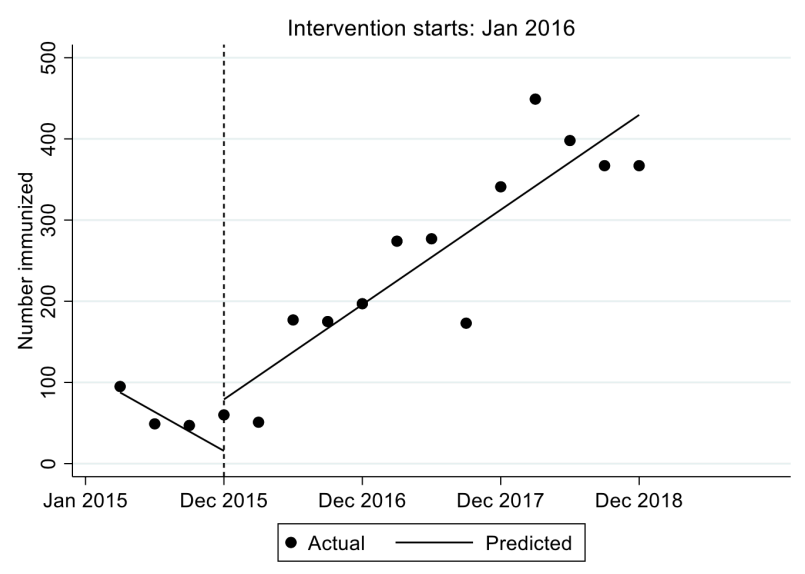

Figure 2. Trend in Immunization attendance.

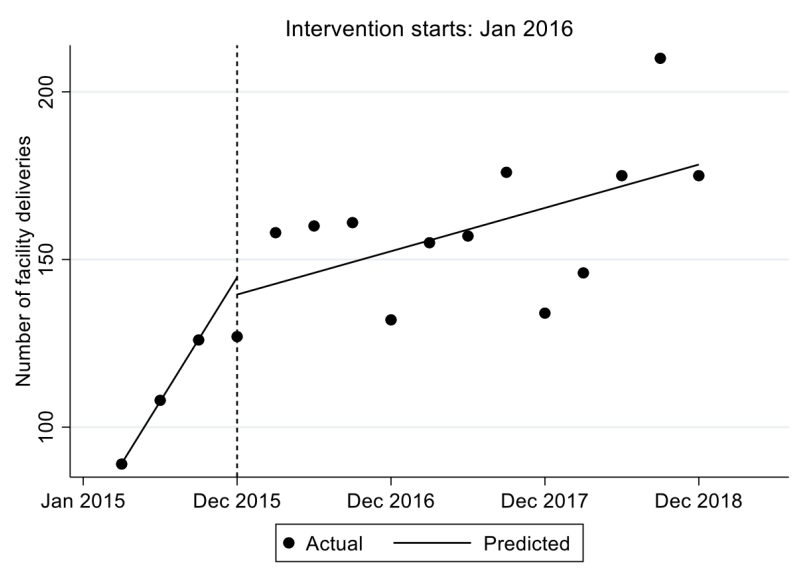

Figure 3. Facility deliveries over the time period.

proportion of women attending ANC, completing ANC visits, referred from community to facility as well as delivering in facility, increased over time. Similarly, Table 3 shows 
Table 2. Trends in outcomes among enrolled pregnant women in Obekai

\begin{tabular}{|c|c|c|c|c|}
\hline & $\begin{array}{l}2015 \\
\text { Baseline } \\
\text { for Obekai }\end{array}$ & 2016 & 2017 & 2018 \\
\hline ANC attendance by pregnant women in the health facilities & $52 \%$ & $88 \%$ & $88 \%$ & $96 \%$ \\
\hline$\%$ of pregnant women completing 4 ANC visits & $29 \%$ & $58 \%$ & $66 \%$ & $76 \%$ \\
\hline Maternal mortality ratio - national & $\begin{array}{l}488 / \\
100,000\end{array}$ & 0 & 0 & 0 \\
\hline Proportion of women satisfied with quality of services at facility & - & $99 \%$ & $100 \%$ & $100 \%$ \\
\hline $\begin{array}{l}\text { Compliance to referrals between the community and primary care facilities } \\
\text {-level } 2\end{array}$ & - & $79 \%$ & $77 \%$ & $89 \%$ \\
\hline $\begin{array}{l}\text { Referrals between the community and primary care facilities level } 2 \\
\text { (denominator is the number of women Identified by } \mathrm{CHW} \text { in the villages)* }\end{array}$ & - & $29 \%$ & $77 \%$ & $59 \%$ \\
\hline $\begin{array}{l}\text { Compliance of referrals between the dispensary level } 2 \text { and County referral } \\
\text { facilities -level } 3\end{array}$ & - & $95 \%$ & $100 \%$ & $100 \%$ \\
\hline $\begin{array}{l}\text { Referrals between } 2016 \text { and } 2018 \text { from level } 2 \text { to levels } 3 \text { facilities } \\
\text { (denominator is the number of enrolled women per year) }^{* *}\end{array}$ & - & $7 \%$ & $5 \%$ & $3 \%$ \\
\hline Facility deliveries & $61 \%$ & $83 \%$ & $86 \%$ & $91 \%$ \\
\hline Home deliveries & $39 \%$ & $17 \%$ & $14 \%$ & $9 \%$ \\
\hline
\end{tabular}

* Increase in referrals from community to dispensary due to the CHWs engagement in the service, later started self-referral

** Reduction in referrals from the dispensaries to level 3 or above due to improved service in the dispensary through health systems implementation

Table 3. Trends in outcomes among children born to enrolled women in Obekai

\begin{tabular}{|c|c|c|c|c|}
\hline & $\begin{array}{l}2015 \\
\text { Obekai }\end{array}$ & 2016 & 2017 & 2018 \\
\hline $\begin{array}{l}\text { Low birth weight and premature babies born in the study population (LBW } \\
\text { currently is about }\end{array}$ & $4.1 \%$ & $7 \%$ & $8 \%$ & $3 \%$ \\
\hline Birth asphyxia & $25 \%$ & $3 \%$ & $3 \%$ & $3 \%$ \\
\hline Neonatal sepsis & $25 \%$ & $1 \%$ & $0 \%$ & $0 \%$ \\
\hline Neonatal mortality & $35 \%$ & $3 \%$ & $2 \%$ & $3 \%$ \\
\hline Fully immunized at 9 months & $6 \%$ & $41 \%$ & $85 \%$ & $90 \%$ \\
\hline Measles immunization coverage at 18 months & $6 \%$ & $0 \%$ & $49 \%$ & $76 \%$ \\
\hline BCG vaccination coverage & $54 \%$ & $81 \%$ & $89 \%$ & $98 \%$ \\
\hline Polio vaccination coverage at birth & $43 \%$ & $90 \%$ & $87 \%$ & $96 \%$ \\
\hline
\end{tabular}

the childhood outcomes over the same period. We observed that there was an improvement in all the childhood indicators over time.

\section{REASONS FOR DELIVERING AT HOME}

In the study, we observed that there was marked improvement in facility delivery with proportion women delivering at home reducing from $39 \%$ in 2015 at baseline to $9 \%$ in 2018 a (91\%) improvement (Table 3). There was still a small proportion who still delivered at home; we interviewed these women to understand why they still opted to deliver at home despite the intervention. Table 4 shows the reasons why women registered in the facility did not deliver in the facility and chose to deliver at home. The main reason being labour starting at night.

\section{DISCUSSION}

This study was conducted as an innovation within the context of the health systems as described by WHO using six pillars ${ }^{1}$ with the objective of linking communities and the primary care facilities. The overall goal was to improve access and utilization of the health care system by communities and to enhance the referral system in the health care delivery system.

Most African countries did not meet the millennium development goals (MDGs) on reducing child mortality, improving maternal health and combating infectious disease (MDGs 4, 5 and 6). Yet, experiences from other continents, as well as recent progress in several countries in the region, prove that the goals can be achieved across Africa. Never- 
Table 4. Reasons for home deliveries in Obekai

\begin{tabular}{|l|l|l|l|l|}
\hline Reason & 2015 Baseline & 2016 & 2017 & 2018 \\
\hline Labour started late at night & - & $9(29 \%)$ & $10(40 \%)$ & $7(40 \%)$ \\
\hline It was raining & - & $5(16 \%)$ & $3(12 \%)$ & $3(18 \%)$ \\
\hline Roads impassable & - & $2(6 \%)$ & $0(0 \%)$ & $0(0 \%)$ \\
\hline Could not get motor cycle rider on time & - & $3(10 \%)$ & $1(4 \%)$ & $2(12 \%)$ \\
\hline Nurses were on strike & - & $2(6 \%)$ & $0(0 \%)$ & $0(0 \%)$ \\
\hline I was alone at home & - & $4(13 \%)$ & $2(8 \%)$ & $1(6 \%)$ \\
\hline No money for private hospital during strike & - & $3(10 \%)$ & $6(24 \%)$ & $2(12 \%)$ \\
\hline Other reasons & - & $3(10 \%)$ & $3(12 \%)$ & $2(12 \%)$ \\
\hline
\end{tabular}

theless, support for rapid scale-up of proven interventions as well as critically needed investments in basic healthcare systems remains insufficient. In most African countries the basic health infrastructure, human resources, equipment and supplies are inadequate to provide essential maternal, child and reproductive health services, and to control and treat infectious diseases. Malaria and other vectorborne diseases that can be controlled and treated continue to take millions of lives throughout Africa and are spreading to more parts of the continent. ${ }^{23}$

The relevance of the health system to maternal and child health can be systematically captured through the building blocks as described by WHO. ${ }^{1}$ An efficient and effective health care system requires a leadership and governance structure, a health care financing system, human resources, a health care delivery system, drugs and equipment and a health information system that is robust and accessible.

This study as implemented has shown improving trends in maternal, child and health care services over the study period. There was a $116 \%, 68 \%$ and $128 \%$ increase in the general outpatient clinic facility attendance, under-five clinic attendance and ANC attendance in 2018 from 2015 compared to the annual projected estimates respectively. This is an indication that the implementation of the health system in the facility by implementing EHC through the FLTR strategy contributed to the improvements. The study as alluded to above was premised on the utilization of the WHO Health Systems pillars through the EHC and FLTR strategies.

These improvements were also evident in an increasing trend in immunization coverage, facility deliveries and reducing home deliveries with attendant high compliance to referrals and reduced referral rates. Similarly, mortality data shows no maternal deaths over the whole period of the study among the over 500 enrolled pregnant women and reduced numbers of neonatal and infant deaths. The neonatal, still birth rates and under-five mortality were observed to be low at $24 / 1000,5.5 / 1000$ and $5.5 / 1000$ respectively compared to $22 / 1000,14.1 / 1000$ and $52 / 1000$ reported in KDHS 2014 respectively nationally. These findings are better than those reported in the area as shown in the results before the onset of the study. The facility deliveries among the women in the study area and the deliveries by skilled health workers at $91 \%$ were higher than reported in the 2014 KDHS of $58.5 \%$ for Busia County and 61\% for Kenya.

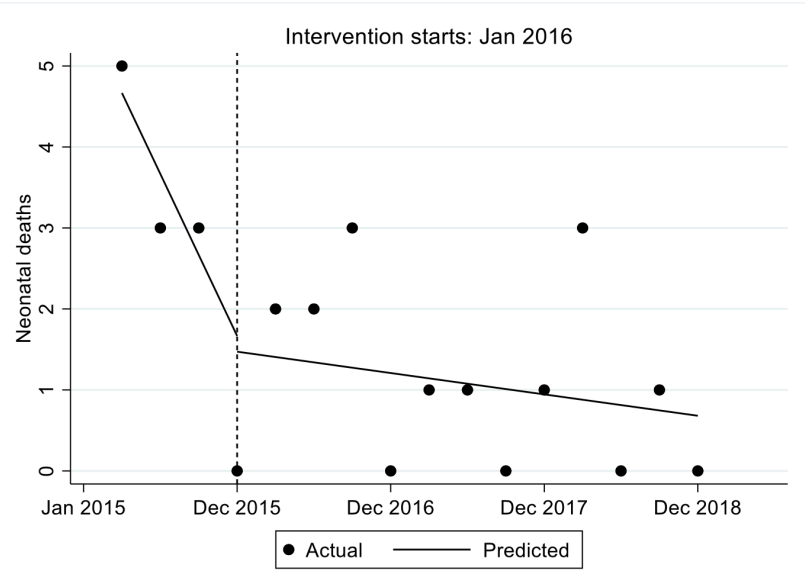

Figure 4. Neonatal deaths over time

The findings of this study on maternal indicators are similar to those found in a study that used the systems approach in maternal health care, Savings Mothers Giving Life (SMGL) project in Uganda and Zambia. ${ }^{24-26}$

In our study the Health System pillars were applied as contextualized below and the trends in the outcomes reported relate to their application.

Leadership and governance have been observed the world over as an important need for the success of any health care system. This is a requirement for the success of the health care delivery system at all level of care (level 1 to 4 in Kenya). In this study the leadership and governance was implemented through the introduction of a clinical officer in a dispensary as the team leader with three nurses. The Research Chair and the research team had monthly supportive supervisory visits in which a standard checklist was used by a team of researchers. There were three pairs of researchers that conducted these monthly visits in which there was a medically trained team member (paediatrician, obstetrician or surgeon) in each pair. The other member in each pair was either a biostatistician or behavioural scientist or health management specialist. This model therefore provided the requisite leadership and governance as provided for by the WHO health systems provisions. This leadership role is expected to be played by the health management teams at county or sub-county level in Kenya or equiv- 
alent in a low- and middle- income country (LMIC).

Healthcare financing is a major hindrance to adequate and satisfactory health care delivery in any country and has been cited as one of the major challenges in developing countries in health care delivery. This component of the health systems pillars was fulfilled through provision of a vote in the study budget for purchase and stocking of drugs and commodities consistent with the Ministry of Health level 2 drug kits for essential drugs and commodities. There was optimal availability of medicines and commodities throughout the study period except for occasional vaccine stock outs since they were solely supplied by the county government.

Service delivery is a complex sub-system for both developed and developing countries. A functional system should aim to provide safe and quality, feasible, affordable and accessible healthcare. Many countries have tried several health service delivery models and hospital reforms without much success and even the most developed countries are still grappling with achieving optimal health care systems. This requires a systematic approach to collect, interpret and use evidence to inform and improve practices within the health system. In this study this was fulfilled by introducing a clinical officer in the dispensary. Normally dispensaries are run by one to three nurses. This model of introducing a clinical officer enabled comprehensive health and clinical care services to be offered in the dispensary and therefore reducing the rate of referrals. The clinical officer provides clinical services that includes minor surgeries and treatment of all ailments for all ages and sex which the nurses are not mandated and trained to do. In addition, we provided a laboratory technician to assist in supporting the clinical officer in diagnosis for common diseases. This reduced the rate of referrals to the health centres and county hospitals (levels 3 or 4 ) for basic diagnostic services.

Medical products, vaccines and technologies are a necessity in a health care system without which utilization slows or is poor and therefore must be procured and made available in health facilities. The provision of enough stocks to complement the County Governments supplies enabled the dispensary to have enough and constant available stocks at all times and therefore the problem of stock outs was reduced. This enabled retention of patients at the level 2 and improved compliance to services and follow up appointments. This also reduced self referrals to levels 3 or 4 facilities by patients.

Health Infrastructure is a crucial sub-system as most health systems in Sub-Saharan Africa are dilapidated and new infrastructure is poorly maintained. A well-functioning health system ensures equitable access to healthcare by ensuring physical infrastructure is available within the required distance, transport infrastructure ensures speedy access and referrals and ICT infrastructure supports timely procurement of supplies and equipment and facilitates effective management of resources. The dispensary had challenges of access from certain villages and we encouraged them to have motorcycle ambulance service from amongst its residents. In light of this a purposely built motorcycle ambulance was procured for the facility towards the end of the study to address the transport and referral challenges. This motorcycle ambulance has all the amenities found in a conventional standard ambulance.

Health management information system (HMIS) is key in a health care delivery system and has to be functional for a successful system of health care. Mobile phone technology and a Medical Records System are important in a health delivery system in order to obtain disease surveillance, demographic and statistical data for planning and follow up. This was operationalised in the dispensary. All the patient records were computerized and data was sent from the field to the central data unit in the dispensary and to the data managers office by the CHWs. This made it easier to identify defaulters and made follow up easier and faster. The default rate was less than $10 \%$, which was mainly due to travel outside the study area.

Human resources are an essential component of a health system and a major shortcoming in sub-Saharan Africa where a deficit of over one million health workers is estimated to exist and Kenya is no exception. There is a direct linear relationship between the density of human resources in health to maternal and child survival. In Kenya the health human resources to population ratio is $13 / 10,000$ populations compared with the WHO figure of $23 / 10,000$ population threshold. 26,27 The dispensary had adequate human resources for health for level 2 facility standards that included a clinical officer, three nurses and a laboratory technician.

This study also addressed the three delays that were outlined by Thaddeus S and Maine D. ${ }^{16}$ The first delay was addressed by education and awareness creation of the need to have a birth preparedness plan by the family by the CHWs and the monthly follow up(WHO Global experience 2010). The second delay was addressed through the birth plan plus the availability of a motorcycle ambulance system in the community. The third delay was addressed by the consistent presence of trained skilled health workers in the dispensary at all times including the constant adequacy of medical products and commodities throughout the 3 years of the study. These personnel received yearly refresher trainings on BeMONC and CeMONC as approved by the Ministry of Health in Kenya. ${ }^{28,29}$ The availability of diagnostic services and basic medical commodities also further addressed this third delay. There was a $100 \%$ satisfaction by patients to the services through an exit survey conducted during the study period.

The findings of this study are therefore testimony to the importance of setting up a health system as provided by the WHO as the driver of universal health coverage through the application of its pillars.

\section{LIMITATIONS OF THE STUDY}

This was pre-post prospective study and the data for the pre-intervention part of the study is only for 2015 and the 2016-2018 constitutes the post intervention period. This does not provide enough data for the pre-intervention period. Data before 2015 is not available at this rural dispensary.

The main limitation in this study was the poor road networks for women to access the facility and means of transport to the facility. By the end of the study a motorcycle (tricyle) ambulance was procured for the dispensary as an in- 
novation. This has improved the clinic attendance, the referral compliance and facility deliveries even at night at the facility.

Despite all the above attempts to implement the best system for universal health coverage (UHC) at the dispensary level, there were still a few home deliveries though at a very reduced level which mainly was either due to too much rain at night or inability to get a companion to go with to the facility.

The other problem encountered over the study period was women who could not attend our clinics and deliver in our intervention facility because of distance. These women were followed up at home as well and were included in our analysis since they complied with the guidelines of the study including attending all antenatal clinics and immunizing their children as required in their chosen facility at their convenience.

\section{CONCLUSIONS}

Trends observed in this study include: i) a significant reduction in maternal and neonatal mortality, ii) increase in fully immunized coverage to over $90 \%$ in 2018 from $21 \%$ in 2015 , increase in ANC attendance of more than 4 visits per pregnancy to over $90 \%$ from $56 \%$, iii) high compliance to referrals and reverse referrals of more than $75 \%$, and iv) high facility deliveries of greater than $91 \%$ from $58.5 \%$ and a reduction in intended home deliveries to $9 \%$ from $41.5 \%$.

These findings indicate that UHC in LMICs should start at the primary care levels where the majority of the population live whether in urban or rural settings. It therefore would imply that enough resources, including human resources, are enhanced in the primary care facilities to attract populations to seek care in these facilities. These facilities should serve all echelons of society irrespective of their social and economic status. There should be a policy shift in the provision of care in which all residents of a country receive health care in these primary care facilities and are then referred to higher level facilities. Universal health care coverage would be difficult to achieve if these facilities are not enabled in human and material resources as a priority.

Acknowledgements: We thank the NACOSTI and IDRC research teams for their careful review of the proposal, Moi University Management for approving the study to be conducted and the staff in our respective departments for pro- viding us time to conduct the study. Special thanks to Prof. Fredrick Were and Prof. Michael English for their advisory role in the advisory committee.

Ethics approval: Written ethics approval to consent to participate was given by the Institutional Research and Ethics Committee of Moi University (IREC) for all participants.

Availability of data and material: The data sets used and analysed during the current study are available from the corresponding author on reasonable request

Funding: This work was carried out with the financial support from the National Commission for Science, Technology and Innovation (NACOSTI) and the International Development Research Centre(IDRC) Canada. The views expressed in this work are those of the creators and do not necessarily represent those of the National Commission for Science, Technology and Innovation, and the International Development Research Centre, Canada or their Board of Governors.The funders did not participate in the design of the study, data collection and its analysis and neither did they participate in the writing of this manuscript.

Authorship contributions: All the authors participated in the development and writing of the manuscript. FOE led the process of manuscript development and writing, EOW provided the guidance and review of the obstetric related issues, AM provided the statistical details and support, DA provided the behavioural aspects, JT provided the community and referral strategy aspects and MN provided the demographic and health systems aspects. All the authors provided edits and reviewed the whole manuscript to its final version as submitted.

Competing interests: The authors completed the Unified Competing Interest form at www.icmje.org/coi_disclosure.pdf (available upon request from the corresponding author), and declare no conflicts of interest.

\section{Correspondence to:}

Prof. Fabian Esamai

Professor of Child Health and Paediatrics

Department of Child Health and Paediatrics

School of Medicine College of Health Sciences

Moi University, Eldoret, Kenya

fesamai2007@gmail.com 


\section{REFERENCES}

1. World Health Organization. Everybody's Business: Strengthening Health Systems to Improve Health Outcomes-WHO's Framework for Action. Geneva: WHO; 2007.

2. United Nations Children's Fund. Committing to Child Survival: A Promise Renewed. Progress Report 2013. http://www.unicef.org/publications/files/APR_P rogress_Report_20139_Sept_2013.pdf.

3. Gilson L. Health Policy and System Research: A Methodology Reader. http://www.who.int/alliance-hp sr/resources/alliancehpsr_reader.pdf?ua=1.2012. Accessed October 4, 2013.

4. McClure EM, Goldenberg RL, Bann CM. Maternal mortality, stillbirth and measures of obstetric care in developing and developed countries. Int J Gynaecol Obstet. 2007;96(2):139-146. doi:10.1016/i.ijgo.2006.1 $\underline{0.010}$

5. Goldenberg RL, McClure EM. Reducing intrapartum stillbirths and intrapartum-related neonatal deaths. Int J Gynaecol Obstet. 2009;107(Suppl 1):S1-3. doi:1 0.1016/j.ijgo.2009.07.014

6. Ahmed I, Ali SM, Amenga-Etego S, et al. Population-based rates, timing, and causes of maternal deaths, stillbirths, and neonatal deaths in south Asia and sub-Saharan Africa: A multi-country prospective cohort study. The Lancet Global Health. 2018;6(12):e1297-e1308. doi:10.1016/s2214-109x(1 8)30385-1

7. Yakoob MY, Ali MA, Ali MU, et al. The effect of providing skilled birth attendance and emergency obstetric care in preventing stillbirths. BMC Public Health. 2011;11(Suppl 3):S7. doi:10.1186/1471-245 8-11-s3-s7

8. Arusei RJ, Ettyang GA, Esamai F. Feeding patterns and growth of term infants in Eldoret, Kenya. Food and Nutrition Bulletin. 2011;32(4):307-314. doi:10.11 77/156482651103200401

9. Ayaya SO, Esamai FO, Rotich J, Olwambula AR. Socio-economic factors predisposing under five-yearold children to severe protein energy malnutrition at the Moi Teaching and Referral Hospital, Eldoret. East Afr Med J. 2004;81:415-421. doi:10.4314/eamj.v81i8.9 $\underline{203}$

10. Kenya National Bureau of Statistics - KNBS - and ICF Macro. Kenya Demographic and Health Survey 2008-09. Calverton, Maryland: KNBS and ICF Macro; 2010 .
11. Kenya National Bureau of Statistics, Ministry of Health/Kenya, National AIDS Control Council/Kenya, Kenya Medical Research Institute, National Council for Population and Development/Kenya, ICF International. Kenya Demographic and Health Survey 2014. Rockville, MD, USA: Kenya National Bureau of Statistics, Ministry of Health/Kenya, National AIDS Control Council/Kenya, Kenya Medical Research Institute, National Council for Population and Development/Kenya, and ICF International; 2015.

12. Goudar SS, Carlo WA, McClure EM, et al. The Maternal and Newborn Health Registry Study of the Global Network for Women's and Children's Health Research. International Journal of Gynecology \& Obstetrics. 2012;118(3):190-193. doi:10.1016/j.ijgo.20 $\underline{12.04 .022}$

13. Government of Kenya. Kenya Service Availability and Readiness Assessment Mapping (SARAM). Nairobi Kenya: Ministry of Health; 2014.

14. McClure EM, Pasha O, Goudar SS, et al. Epidemiology of stillbirth in low-middle income countries: A Global Network Study. Acta Obstetricia et Gynecologica Scandinavica.

2011;90(12):1379-1385. doi:10.1111/j.1600-0412.201 1.01275.X

15. Countdown Working Group on Health Policy and Health Systems, Cavagnero E, Daelmans B, Gupta N, Scherpbier R, Shankar A. Assessment of the health system and policy environment as a critical complement to tracking intervention coverage for maternal, newborn, and child health. The Lancet. 2008;371(9620):1284-1293. doi:10.1016/s0140-6736(0 8)60563-2

16. Thaddeus S, Maine D. Too far to walk: Maternal mortality in context. Social Science \& Medicine. 1994;38(8):1091-1110. doi:10.1016/0277-9536(94)902 $\underline{26-7}$

17. Kenya National Bureau of Statistics. Kenya Demographic and Health Survey 2003. Nairobi: Government of Kenya; 2004.

18. Government of Kenya. Kenya Health Policy Framework 2012 - 2030. Nairobi: Government of Kenya; 2012.

19. Government of Kenya. The Kenya Vision 2030. Nairobi: Government of Kenya; 2010.

20. Government of Kenya. The National Health Sector Strategic Plan III, 2013-2017. Nairobi: Government of Kenya; 2012. 
21. Esamai F, Nangami M, Tabu J, Mwangi A, Ayuku D, Were E. A system approach to improving maternal and child health care delivery in Kenya: Innovations at the community and primary care facilities (a protocol). Reprod Health. 2017;14(1). doi:10.1186/s12 978-017-0358-6

22. Mwangi A, Nangami M, Tabu J, Ayuku D, Were E, Fabian E. A system approach to improving maternal and child health care delivery in Kenyan communities and primary care facilities: Baseline survey on maternal health. Afr H Sci. 2019;19(2):1841. doi:10.43 14/ahs.v19i2.6

23. World Health Organization. MDG Report by African Steering Group. Geneva: WHO; 2008.

24. Florina S, Lawrence M, Jessica H, Jonathan L, Reeti H, Marta L, et al. Glob Health Sci Pract. 2019;7(suppl 1):S6-S26. doi:10.9745/GHSP-D-18-004 $\underline{27}$
25. Quam L, Achrekar A, Clay R. Saving Mothers, Giving Life: A Systems Approach to Reducing Maternal and Perinatal Deaths in Uganda and Zambia. Glob Health Sci Pract. 2019;7(suppl 1):S6-S26. doi:10.9745/GHSP-D-19-00037

26. Bhutta ZA, Lassi ZS, Pariyo G, Huicho L. Global Experience of Community Health Workers for Delivery of Health Related Millennium Development Goals: A Systematic Review, Country Case Studies, and Recommendations for Integration into National Health Systems. Geneva: Global Health Workforce Alliance, World Health Organization; 2010.

27. World Health Organization. Global Atlas of Health Work Force. Geneva: WHO; 2010.

28. Kenya Ministry of Health. Basic Paediatric Protocols 2016. Nairobi Kenya: Kenya Ministry of Health; 2016.

29. Kenya Ministry of Health. National Guidelines for Quality Obstetrics and Perinatal Care and National Guidelines on Essential Newborn Care in Kenya. Nairobi Kenya: Kenya Ministry of Health; 2011. 